\title{
Design and Analysis of Elliptical Nozzle in AJM Process using Computational Fluid Dynamics
}

\author{
Baranitharan $\mathbf{P}^{1^{*}}$ and Zeelan Basha $\mathbf{N}^{2}$ \\ ${ }^{1}$ Department of Mechanical Engineering, Sree Sakthi Engineering College, Karamadai, Coimbatore, \\ Tamilnadu, India \\ ${ }^{2}$ Department of Mechanical Engineering, College of Engineering and Technology, Post Box No: 395, \\ Wollega University, Nekemte, Ethiopia
}

\begin{tabular}{lll}
\hline & \multicolumn{1}{c}{ Abstract } & Article Information \\
\hline Abrasive jet machining (AJM) is a micromachining process, where material is removed from & Article History: \\
the work piece by the erosion effect of a high speed stream of abrasive particles carried in a & Received : 10-01-2015 \\
gas medium, which are emerging from a nozzle. Abrasive machining includes grinding super & Revised $: 22-03-2015$ \\
finishing honing, lapping polishing etc. The common nozzle shape presently used in AJM & Accepted :24-03-2015 \\
\cline { 2 - 2 } machining process is rectangle and circular shape nozzle which gives a low flow rate and & Keywords: \\
further demands on decreasing the material removal rate (MRR), so this research mainly & AJM Process \\
focuses on designing nozzle geometry to improve flow rate and MRR in AJM machining & Elliptical Nozzle \\
process. The elliptical shape nozzle has been designed and analyzed using computational & Flow rate \\
fluid dynamics software (CFD). CFD is the most efficient software for flow rate analysis. The & CFD \\
\cline { 2 - 2 } result shows the improvement in flow rate about 574.2m/sec and MRR of newly designed & Baranitharan P Author: \\
nozzle geometry i.e elliptical shape in abrasive jet machining. & E-mail: \\
Copyright@2015 STAR Journal, Wollega University. All Rights Reserved. & baranipsivam@gmail.com \\
\hline
\end{tabular}

\section{INTRODUCTION}

Abrasive water jets, namely water jets containing abrasive particles, have a considerable niche in the material processing industry. Like laser cutting instruments they are accurate, easily managed and cause very little loss of material. However, abrasive jet cutting does not involve high temperatures, which is characteristic to laser cutting, and as a result they are suitable for practically any material. Furthermore, the instrumentation required for high-speed jets is simpler and much cheaper. Consequently, jet cutting can be implemented in a broad range of industries, ranging from small machine shops and quarries, to large sheet metal, composites or ceramic processing in the car and aircraft industries (Umang Anand et al., 2003).

Abrasive Jet Micro Machining (AJMM) is an unconventional machining technique, in which high pressure gas is used as medium to accelerate the abrasive grits and make them form an effect of impact or erosion on workpiece. It's applicable to the micro structural machining and the surface treatment of parts. The principle of Abrasive Jet Machining (AJM) is mostly analyzed and a set of principle experiment device is contrived, the AJM process is observed through experiments, the characteristics of AJM are analyzed (Yan Qiusheng et al., 2002). This paper presents CFDsimulations of different diffuser elements used in valveless diffuser pumps and nozzle elements used in dynamic micropumps. The results are compared with measurements and with analytic expressions based on empirical results known from basic fluid mechanics. The working principles of the diffuser element in the valve-less diffuser pump and the nozzle-element in the dynamic micropump are investigated (Olsson et al., 1997).

Machining process is the common method of production process. Development of newer materials wants to change traditional to non-traditional machining methods. Cutting of brittle material by conventional machining is difficult than unconventional machining process (Sehgal, 2011). Now a day's CFD is the most famous software for flow analysis (Alina Oancea et al., 2012). The existing circle and rectangle shape nozzle gives a low flow rate which further demands on decreasing the material removal rate (Bhaskar Chandra, 2011). The traditional methods used for machining processes like cutting and deburring can be efficiently replaced by Abrasive Jet Machine. Hard and Brittle materials can be efficiently cut by using this technique. In the present work an experimental investigation of cutting the ceramic tiles with abrasive jet machining is carried out and the effect of different cutting parameters on the Material Removal rate is studied (Gulhane et al., 2013)

The literature study reveals that the process was developed a few decades ago, many experiments and studies on this process has been conducted and there is still scope for research and development. 


\section{MATERIALS AND METHODS}

The major field of application of AJM process is in the machining of essentially brittle materials and heat sensitive materials like glass, quartz, sapphire, semiconductor materials, mica and ceramics. It is also used in cutting slot, thin sections, countering, drilling, deburring, for producing integrate shapes in hard and brittle materials. It is often used for cleaning and polishing of plastics nylon and Teflon components. Delicate cleaning, such as removal of smudges from antique documents, is also possible with AJM.

\section{Circular Nozzle}

The abrasive particles are directed into the work surface at high velocity through nozzles. Therefore, the material of the nozzle is subjected to great degree of abrasion wear and hence these are made of hard materials such as tungsten carbide or synthetic sapphire. Tungsten carbide nozzles are used for circular cross-sections in the range of $0.12-0.8 \mathrm{~mm}$ diameter, for rectangular sections of size $0.08 \times 0.05$ to $0.18 \times 3.8 \mathrm{~mm}$ and for square sections of size up to $0.7 \mathrm{~mm}$. Sapphire nozzles are made only for circular cross-sections. The size varies from 0.2 to $0.7 \mathrm{~mm}$ diameter. Nozzles are made with an external taper to minimize secondary effects due to ricocheting of abrasive particles. Nozzles made of tungsten carbide have an average life of 12 to 30 hours while nozzles of sapphire last for about 300 hour of operation when used with $27 \mu \mathrm{m}$ abrasive powder. The rate of material removal and the size of machined area are influenced by the distance of the nozzle from the work piece. The abrasive particles from the nozzle follow a parallel path only for a short distance and then the jet flares resulting in the over sizing of the hole. It is observed that the jet stream is initially a cylinder for about $1.6 \mathrm{~mm}$ and then it flares into a cone of $7^{\circ}$ included angle. The material removal rate initial increases with increase in the distance of the nozzle from the work piece because of the acceleration of particles leaving nozzle. This increase is maximum up to a distance about $8 \mathrm{~mm}$ and then it steadily drops off because of increase in machining area for the same amount of abrasive and decrease in velocity of abrasive stream due to drag.

\section{Circular Shape Nozzle}

The circular nozzle has Low material removal rate and hence application is limited. It has a narrow flow path from the outlet section (figure 1). Nozzles edges will get etched by the mixture of abrasive particles and air. After the optimum value the diameter diverge by that the life of nozzle is calculated.

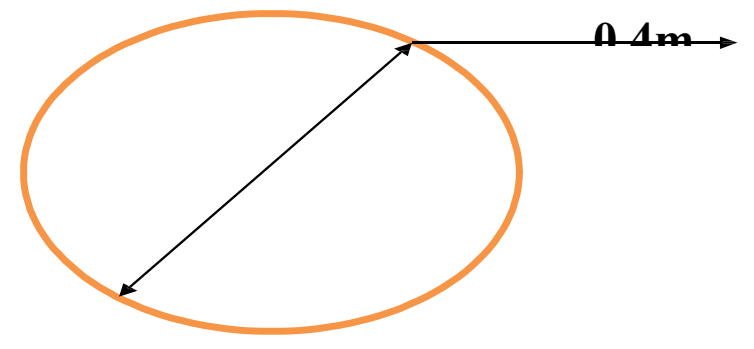

Figure 1: Existing Circular Nozzle Design

\section{Specification of Circular Nozzle}

The table 1 shows the specification of circular nozzle for the development and analyzing of flow rate and velocity
Table 1: specification of circular nozzle

\begin{tabular}{ll}
\hline Medium & Air , CO2 ,N2 \\
Abrasive & Sic, Al2O3 (of size $20 \mu$ to $50 \mu$ ) \\
Flow rate of abrasive & 3 to $20 \mathrm{gram} / \mathrm{min}$ \\
Velocity & 150 to $300 \mathrm{~m} / \mathrm{min}$ \\
Pressure & 2 to $8 \mathrm{~kg} / \mathrm{cm} 2$ \\
Nozzle size & 0.07 to $0.40 \mathrm{~mm}$ \\
Material of nozzle & WC, Sapphire \\
Nozzle life & 12 to $300 \mathrm{hr}$ \\
Standoff distance & 0.25 to $15 \mathrm{~mm}$ (8mm generally) \\
& Non Metals like glass, ceramics, and \\
Work material & granites.Metals and alloys of hard \\
& materials likegermanium, silicon etc \\
part application & Drilling, cutting, deburring, cleaning \\
\hline
\end{tabular}

\section{Modification of Nozzle Geomentry}

Designed the elliptical nozzle for required dimension is drawn shown in figure 2. Design of modified nozzle shape using CFD has been analyzed and the table 2 shows the improvement in characteristics. To examine analysis are used various nozzle shapes and among those design required and designed shapes is obtained. From that nozzle the output is taken for need.

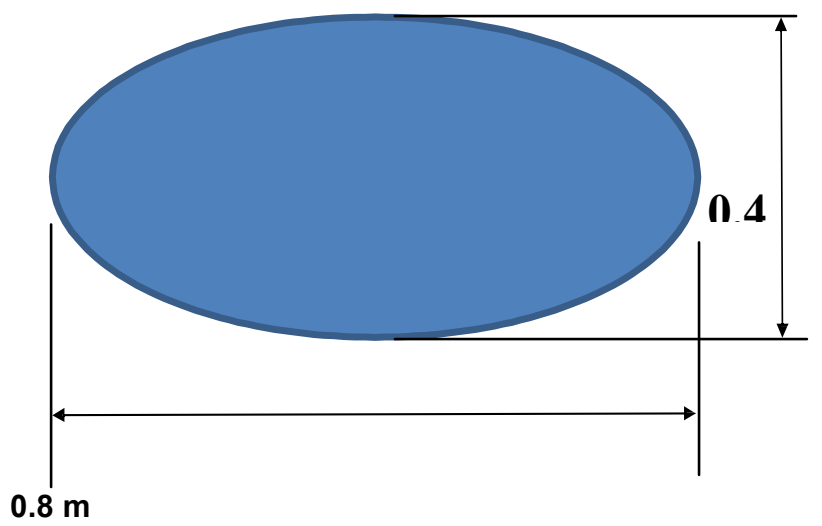

Figure 2: Elliptical Nozzle Design Dimension

Table 2: Experimental Parameters in AJM -Elliptical Nozzle

\begin{tabular}{clc}
\hline No & \multicolumn{1}{c}{ AJM Parameter } & Condition \\
\hline 1 & Type of medium & Air \\
2 & Velocity & $190 \mathrm{~m} / \mathrm{sec}$ \\
3 & Jet pressure & $2-3 \mathrm{k} \mathrm{g} / \mathrm{cm} 2$ \\
4 & Meshing level & 3 \\
5 & Meshing gap size & $0.0008 \mathrm{~mm}$ \\
6 & Analysis type & internal flow \\
\hline
\end{tabular}

Experiments Parameters in Abrasive Jet Machining Process in Elliptical Nozzle (table 3 ) considered as Air is medium, Velocity $19 \mathrm{~m} / \mathrm{sec}$ and Abrasive jet pressure fixed 2-3 kg/cm2. Meshing level and Meshing gap size are 3 and $0.0008 \mathrm{~mm}$ accordingly.

\section{RESULT AND DISCUSSION \\ Shape of Design-Circular Nozzle}

Circular Nozzle- Velocity: The figure 3 shows the flow is from $Y$ to $X$ direction and the initial velocity is $61.559 \mathrm{~m} / \mathrm{s}$. Obtained the final velocity is $603.535 \mathrm{~m} / \mathrm{s}$ in circular shaped nozzle according the parameters fixed. 


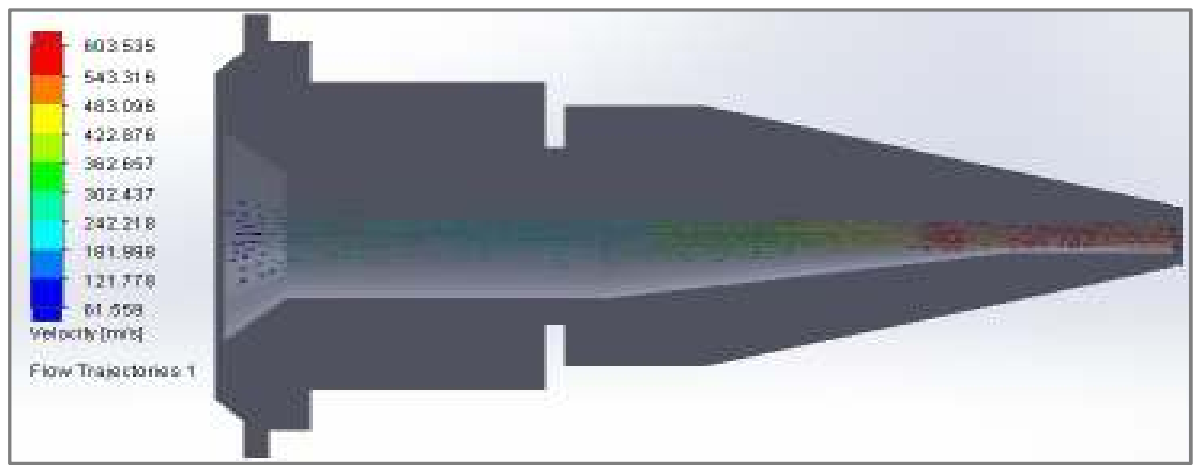

Figure 3: Velocity of circular nozzle

Circular Nozzle -Pressure: Figure 4 shows the assigned outlet pressure of 2 bar in environment pressure. 6.97 e10 is inlet pressure of the nozzle and finally obtained nozzle velocity 2.83 e13.



Figure 4: Pressure of circular nozzle

Circular Nozzle -Meshing: Figure 5 shows the meshing process, where meshing has done for circular nozzle at a level of four to find the analysis result accurate.

Circular Nozzle -Velocity Analysis: The Figure 6 shows analysis result for velocity and pressure, The velocity has been analyzed and values achieved from 0 to $612.8 \mathrm{~m} / \mathrm{s}$. The result has been considered for comparison of modified elliptical nozzle to know the improvement in design modification.



Figure 5: Meshing of circular nozzle

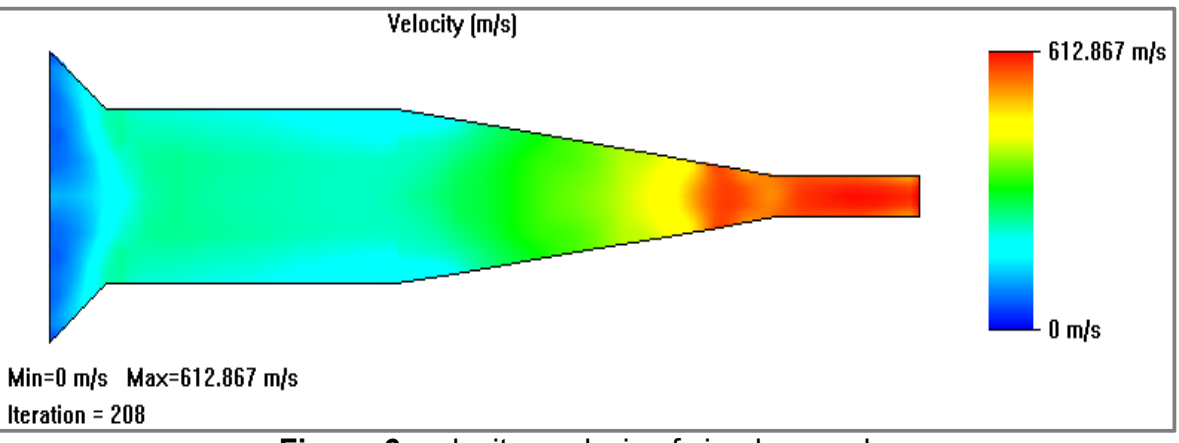

Figure 6: velocity analysis of circular nozzle 
Baranitharan and Zeelan Basha

\section{Circular Nozzle -Pressure Analysis}

The figure 7 shows analysis result for pressure, The pressure has been analyzed from minimum value $6.9690 \mathrm{e}+010$ pa to maximum value $2.83365 \mathrm{e}+013$ pa.the
Sci. Technol. Arts Res. J., Jan-March 2015, 4(1): 171-179

result has been considered for comparison of modified elliptical nozzle to know the improvement in design modification.

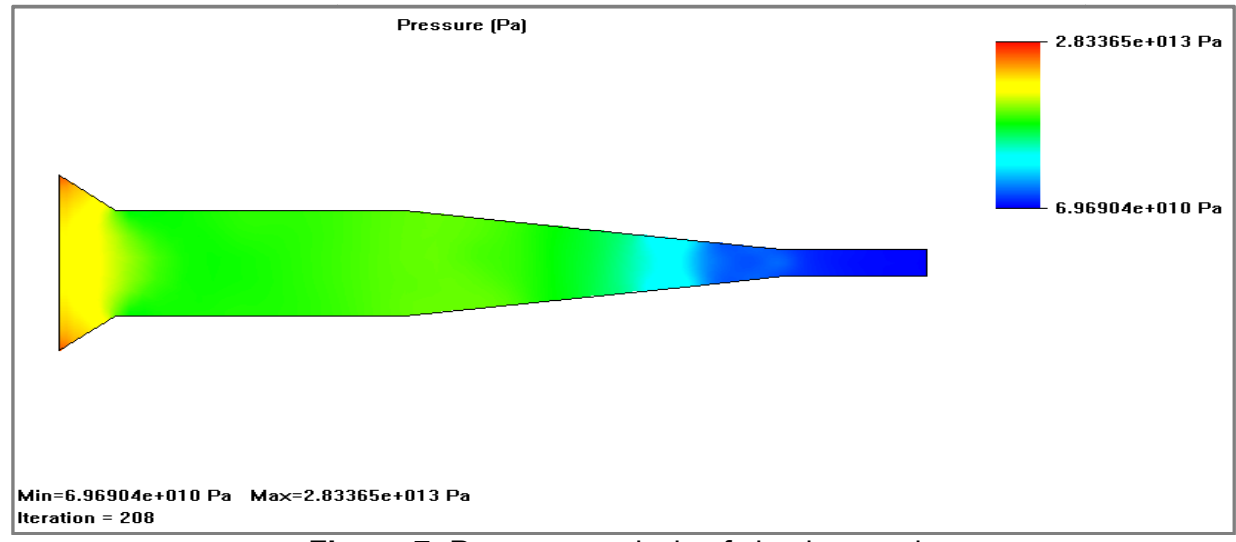

Figure 7: Pressure analysis of circular nozzle

Specification: Meshing size -4 and Inlet velocity- 190 $\mathrm{m} / \mathrm{s}$ Environment pressure -2 bar

Surface Parameters: The table 3 shows the analysis result of circular nozzle surface parameters, The local and integral parameters of surface parameters has been analyzed using CFD to obtain the activity of performance. The result has been considered for comparison of modified elliptical nozzle to know the improvement in design modification.

Table 3: Surface Parameters of Circular Nozzle using CFD

\begin{tabular}{|c|c|c|c|c|c|}
\hline Parameter & Minimum & D.Saximum & Average & Bulk Averago & [Surface Area lmin'] \\
\hline Dansity [kp/m³] & 1DED310.99 & 413856402 & 3409164.13 & 351816.587 & $4.91314 E=07$ \\
\hline Velocity lms] & 402.860913 & 605.555532 & 559.154105 & 587.295578 & $4.91314 E-07$ \\
\hline Velocity $\left(\mathrm{C}^{\prime}\right)$ [m/s] & -36.662426 & 44.8987155 & 3.31046491 & 3.83955603 & $=07$ \\
\hline Velocity (Z) [m/s] & -51.2011606 & 46.166198s & -1.15451074 & -0.049732174 & $4.91314 E-07$ \\
\hline Mach Number [1] & 1.32896398 & 2.66340609 & 2.26069984 & 2.32795836 & $4.91314 E-07$ \\
\hline
\end{tabular}

\begin{tabular}{|c|c|c|c|c|}
\hline Value & X-component & $Y$-component & Z-component & Surface Area [m²] \\
\hline-0.000240711 & 6 & 0 & 0 & $4.91314 E-07$ \\
\hline $4.91316 E=07$ & $=4.91314 \mathrm{E}-07$ & $-4.6 E-424 E=21$ & $2.12872 \mathrm{E}=20$ & $4.91314 \mathrm{E}=07$ \\
\hline $0.95-5.5376$ & 0 & 0 & 0 & $4.91314 \mathrm{E}=07$ \\
\hline $5,02655=07$ & Q & 0 & की & $5,02655 \mathrm{C}=07$ \\
\hline
\end{tabular}

In circular nozzle, obtained velocity of $559.15 \mathrm{~m} / \mathrm{s}$ and the mass flow rate is $-853.87 \mathrm{~kg} / \mathrm{s}$ and also in case of necessity the volume and temperature can be obtained through the above data's.
Graph for Circular Nozzle-Velocity: The graph has been plotted between velocity $\mathrm{m} / \mathrm{s}$ and number of iteration(figure 8). The graph shows that the velocity decrease from 670 to $570 \mathrm{~m} / \mathrm{s}$ with increase of number of iteration from -100 to 210 in circular nozzle of AJM process

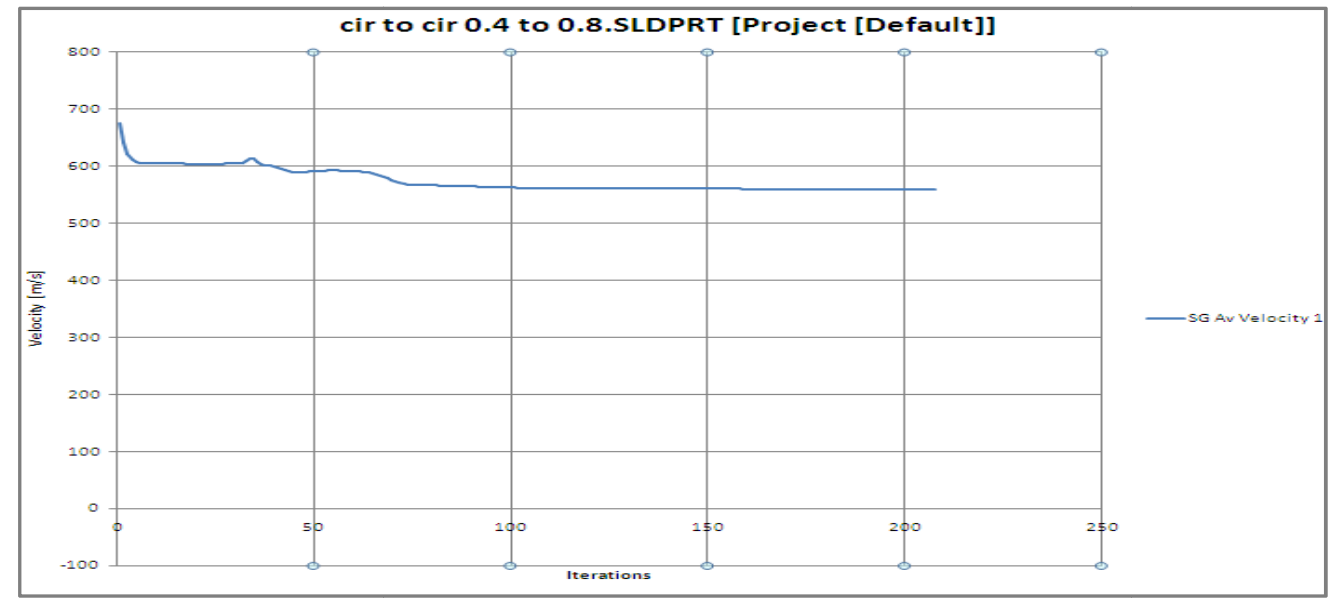

Figure 8: Circular nozzle the velocity was taken with iteration 
Baranitharan and Zeelan Basha

Graph For Circular Nozzle -Volume Flow Rate: The graph has been plotted between volume flow rate $\mathrm{m}^{3} / \mathrm{s}$ and number of iteration (figure 9). The graph shows that
Sci. Technol. Arts Res. J., Jan-March 2015, 4(1): 171-179

the volume flow rate increases from -0.000475 to $0.00025 \mathrm{~m}^{3} / \mathrm{s}$ with increase of number of iteration from 0 to 210 in circular nozzle of AJM process

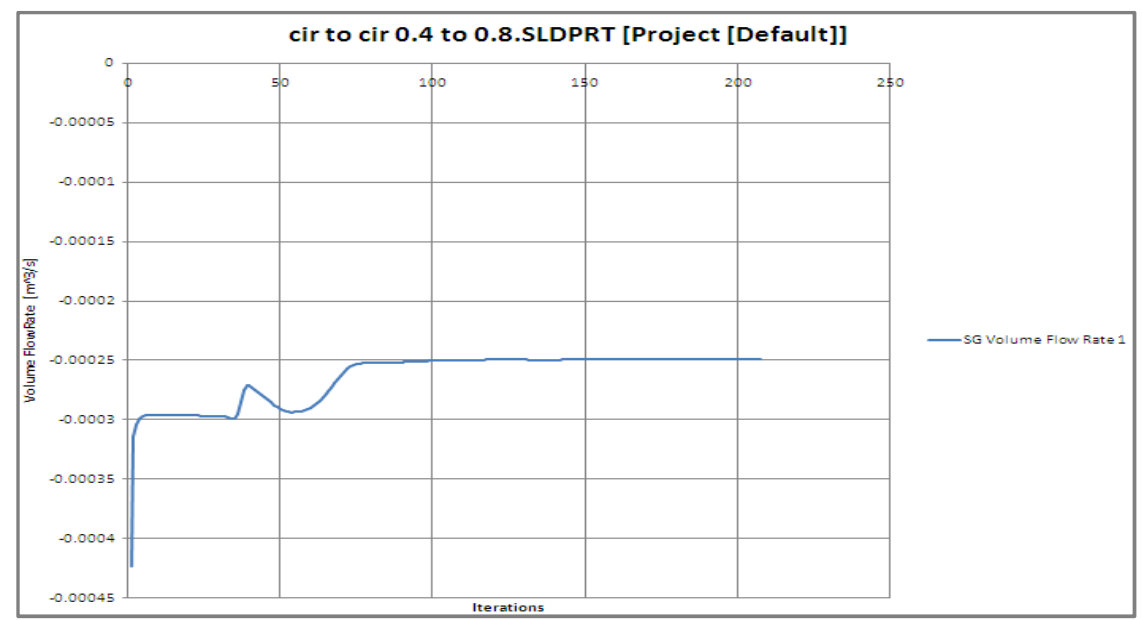

Figure 9: Circular nozzle the volume flow rate was taken with iteration

Graph For Circular Nozzle -Mass Flow Rate: The graph has been plotted between mass flow rate $\mathrm{kg} / \mathrm{s}$ and number of iteration (figure 10). The graph shows that the mass flow rate decrease from 0 to $-870 \mathrm{~kg} / \mathrm{s}$ with increase of number of iteration from 150 to 210 in circular nozzle of AJM process

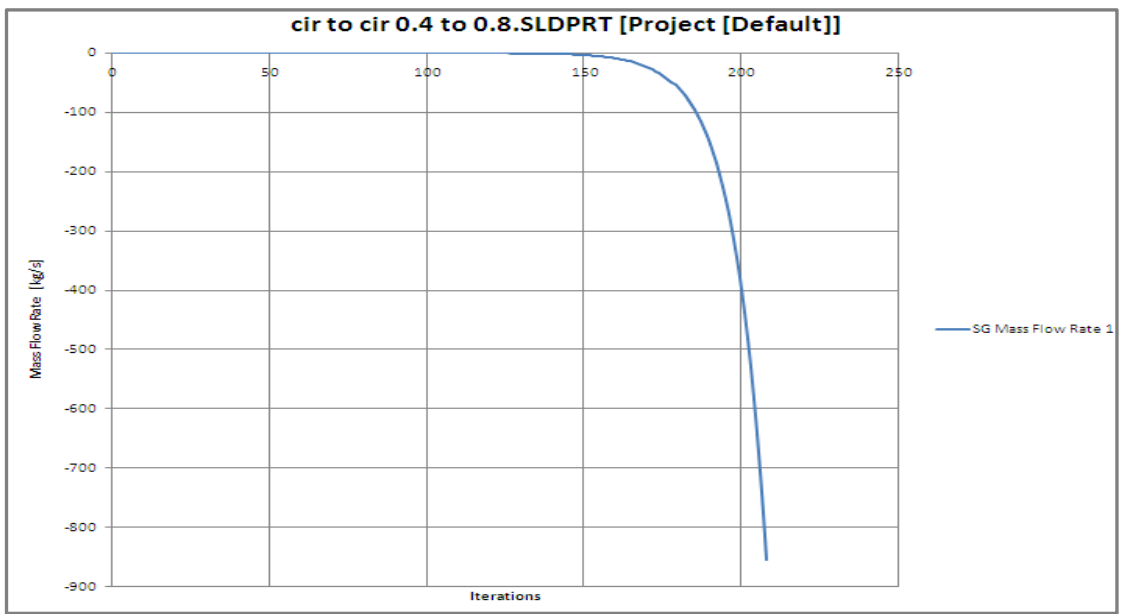

Figure 10: Circular nozzle the mass flow rate was taken with iteration

Graph For Circular Nozzle -Static Pressure: The graph has been plotted between static pressure pa and number of iteration (figure 11). The graph shows that the static pressure from $-1 \mathrm{E}+11$ to $-1.5 \mathrm{E}+11$ pa with increase of number of iteration from 145 to 210 in circular nozzle of AJM process.

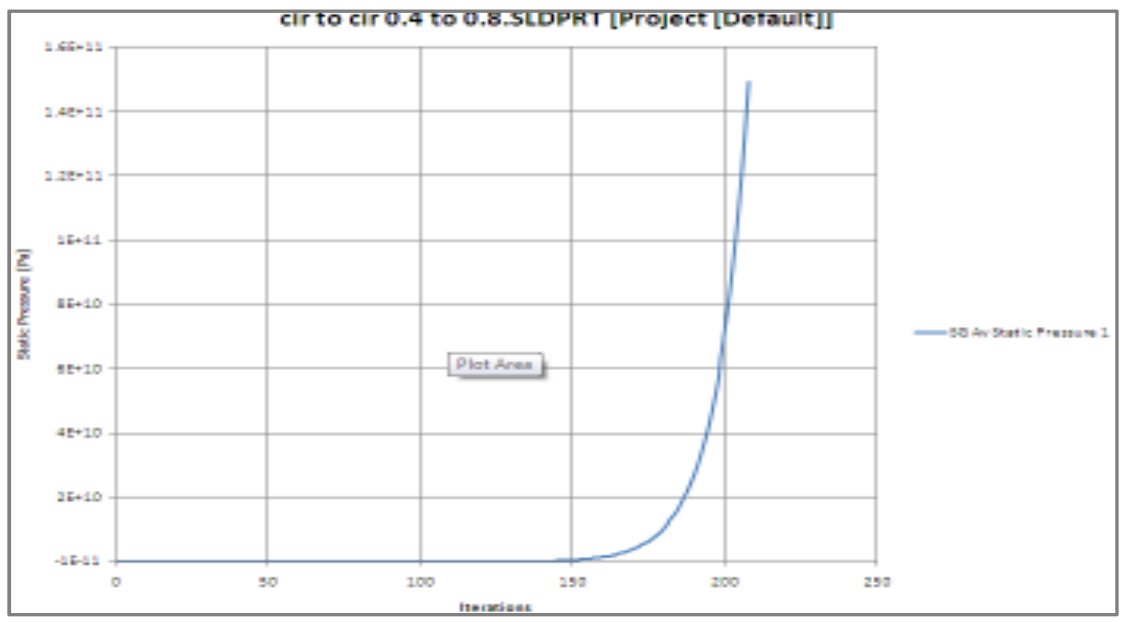

Figure 11: Circular nozzle the static pressure was taken with iteration 
Baranitharan and Zeelan Basha

Elliptical Nozzle-Velocity: The Figure 12 shows the flow is from $Y$ to $X$ direction and the initial velocity of 64.196 $\mathrm{m} / \mathrm{s}$ and we obtain the final outlet velocity is $649.26 \mathrm{~m} / \mathrm{s}$ in elliptical shaped nozzle.
Sci. Technol. Arts Res. J., Jan-March 2015, 4(1): 171-179

Elliptical Nozzle-Pressure: The figure 13 shows the assigned the outlet pressure 2 bars in environment pressure.1.39 e11 is inlet pressure of the nozzle and finally obtained nozzle velocity $1.96 \mathrm{e} 13$.

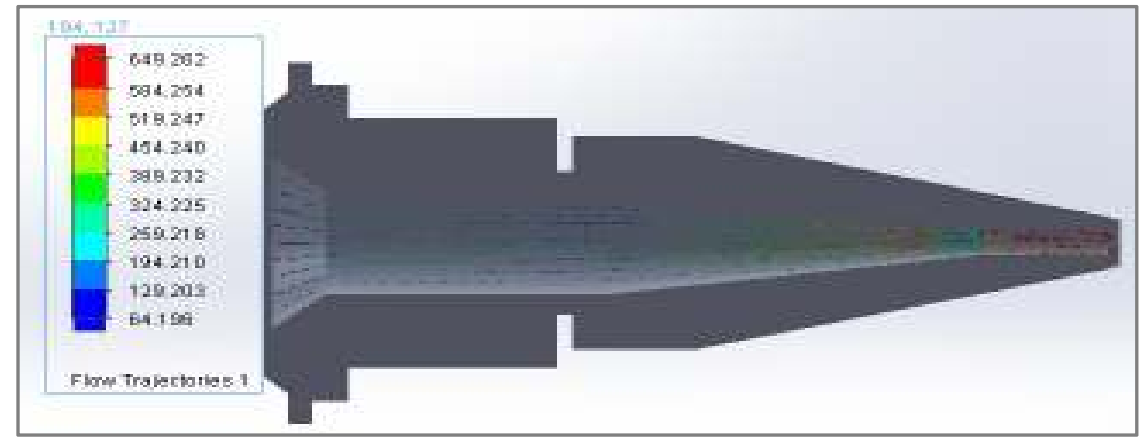

Figure 12: Velocity of Elliptical nozzle

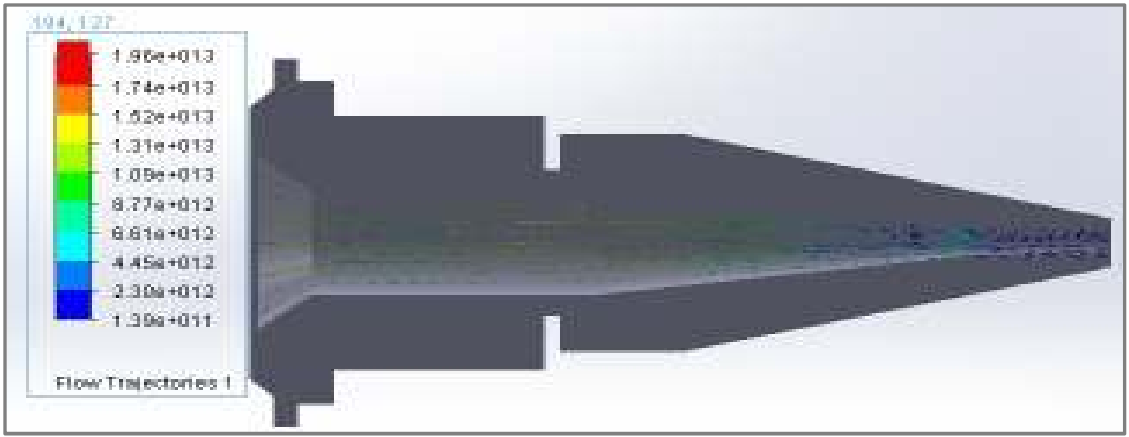

Figure 13: Pressure of Elliptical nozzle

Elliptical Nozzle-Meshing: The figure 14 shows is a meshing process, where seshing has done for elliptical nozzle at a level of four to find the analysis accuracy of result.

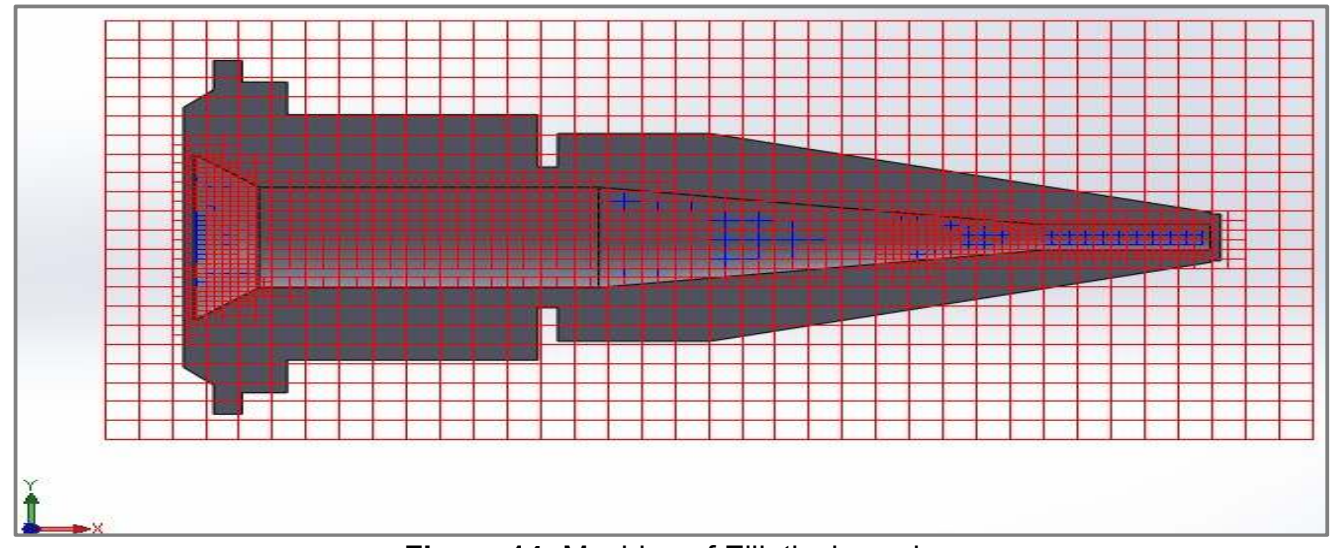

Figure 14: Meshing of Elliptical nozzle

Analysis Of Elliptical Nozzle-Velocity: The figure 15 shows analysis result for velocity, the velocity has been analyzed from 0 to $612.8 \mathrm{~m} / \mathrm{s}$. The result has been

considered for comparison of circular nozzle and modified design elliptical nozzle to know the improvement in design modification

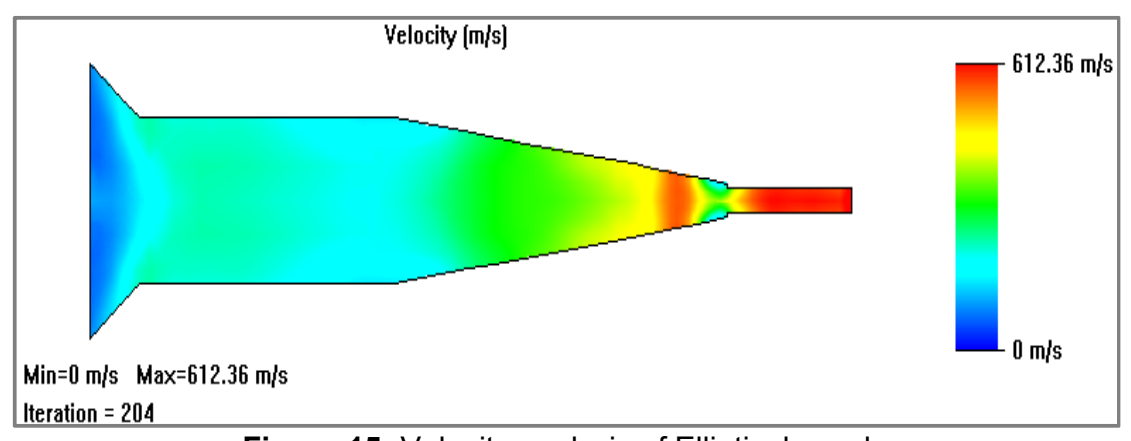

Figure 15: Velocity analysis of Elliptical nozzle 


\section{Baranitharan and Zeelan Basha}

Analysis of Elliptical Nozzle-Pressure: The figure 16 shows analysis result for pressure, The pressure has been analyzed and values achieved from minimum value $6.9690 \mathrm{e}+010 \mathrm{pa}$ to maximum value $2.83365 \mathrm{e}+013 \mathrm{pa}$ in circular nozzle and also the pressure has been analyzed and values achieved from minimum value $1.95593 e+013$
Sci. Technol. Arts Res. J., Jan-March 2015, 4(1): 171-179

pa to maximum value $1.39151 \mathrm{e}+011 \mathrm{pa}$ in elliptical nozzle .The circular and elliptical nozzle result has been considered for comparison to know the improvement in design modification. The result revealed that the modified design elliptical model has improvement in output nozzle pressure.



Figure 16: Pressure analysis of Elliptical nozzle

Specification: 1 Meshing size -4 and Inlet velocity- 190 $\mathrm{m} / \mathrm{s}$ Environment pressure -2 bar.

Comparison of Nozzle -Surface Parameters: The table 4 shows the analysis result of elliptical nozzle surface parameters, The local and integral parameters of surface parameters has been analyzed using CFD to obtain the activity of performance. The result has been considered for comparison of modified elliptical nozzle to know the improvement in design modification. The result revealed improvement in mass flow rate, pressure and velocity in elliptical nozzle.

Table 4: Surface Parameters of Elliptical Nozzle using CFD

Local parameters

\begin{tabular}{|c|c|c|c|c|c|}
\hline Parameter & Minimum & Maximum & Average & Bulk Average & Surface Area [m/2] \\
\hline Pressure [Pa] & $1.39151 \mathrm{E}+11$ & $1.69457 \mathrm{E}+11$ & $1.55093 \mathrm{E}+11$ & $1.5569 E+11$ & $3.6824 \mathrm{E}-07$ \\
\hline Density [kg/m³] & 2316868.16 & 4128746.64 & 3694354.34 & 3741823.63 & $3.6824 \mathrm{E}-07$ \\
\hline Velocity [m/s] & 452.982531 & 598.243604 & 574.626536 & 577.788986 & $3.6824 \mathrm{E}-07$ \\
\hline Velocity $(\mathrm{X})[\mathrm{m} / \mathrm{s}]$ & 451.776253 & 598.197636 & 573.833821 & 577.03568 & $3.6824 \mathrm{E}-07$ \\
\hline Velocity $(Y)$ [m/s] & -48.8137871 & 19.1989013 & -16.0572888 & $-15,750205$ & $3.6824 \mathrm{E}-07$ \\
\hline Velocity $(Z)[\mathrm{m} / \mathrm{s}]$ & -21.3051564 & 26.5309842 & -1.12959586 & -1.04557869 & $3.6824 \mathrm{E}-07$ \\
\hline Aach Number [] & 1.57094857 & 2.59175731 & 2.38453212 & 2.40811126 & $3.6824 \mathrm{E}-07$ \\
\hline Temperature (Fluid) $[\mathrm{K}]$ & 133.049423 & 206.997301 & 146.245128 & 144.605965 & $3.6824 \mathrm{E}-07$ \\
\hline $\begin{array}{l}\text { Relative Pressure [Pa] } \\
\text { intagrel paramaters }\end{array}$ & $1.39151 \mathrm{E}+11$ & $1.69457 \mathrm{E}+11$ & $1.55092 \mathrm{E}+11$ & $1.5569 \mathrm{E}+11$ & $3.6824 \mathrm{E}-07$ \\
\hline Parameter & Value & $x-60$ & nponent & Z-compothen: & Surface Area [mn 2] \\
\hline Mass Flow Raie [kg/s] & -712.8323 & 2 & 0 & 을 & $3.8824 \mathrm{E}-07$ \\
\hline Volume Flow Rate [m 3/3] & -0.00019213 & & & 0 & $3.6824 \mathrm{E}-07$ \\
\hline Surface Area $\left[\mathrm{m}^{2} \mathrm{z}\right]$ & $3.6824 E=077$ & $-3.68 .24 \mathrm{E}-07$ & $-1.69219 \equiv-21$ & $2.36361 \mathrm{E}-21$ & $3.6824 E-07$ \\
\hline Tulal Entilalory Rale [W] & 225463361 & 의 & 0 & 0 & $3.6824 E$ O 7 \\
\hline Uniformity Index [] & 0972016809 & 문 & a & D & $3.8824 E-07$ \\
\hline CAR Fund area $\left(\mathrm{m}^{-} \mathrm{z}\right)$ & $376724 t-51$ & & & & 3. $7607241-007$ \\
\hline
\end{tabular}

The table 4 shows the obtained velocity is $559.15 \mathrm{~m} / \mathrm{s}$, and the mass flow rate is $-853.87 \mathrm{~kg} / \mathrm{s}$ and also in case of necessity the volume and temperature also can be obtained. In elliptical nozzle (table 4 ) the velocity obtained $574.62 \mathrm{~m} / \mathrm{s}$ and the mass flow rate is $-721.62 \mathrm{~kg} / \mathrm{s}$, which shows the $5 \%$ improvement of mass flow rate in elliptical nozzle
Graph for Elliptical Nozzle-Velocity: The graph has been plotted between velocity $\mathrm{m} / \mathrm{s}$ and number of iteration (figure 17). The graph shows that the velocity decrease from 700 to $580 \mathrm{~m} / \mathrm{s}$ with increase of number of iteration from -100 to 210 in elliptical nozzle of AJM process. 


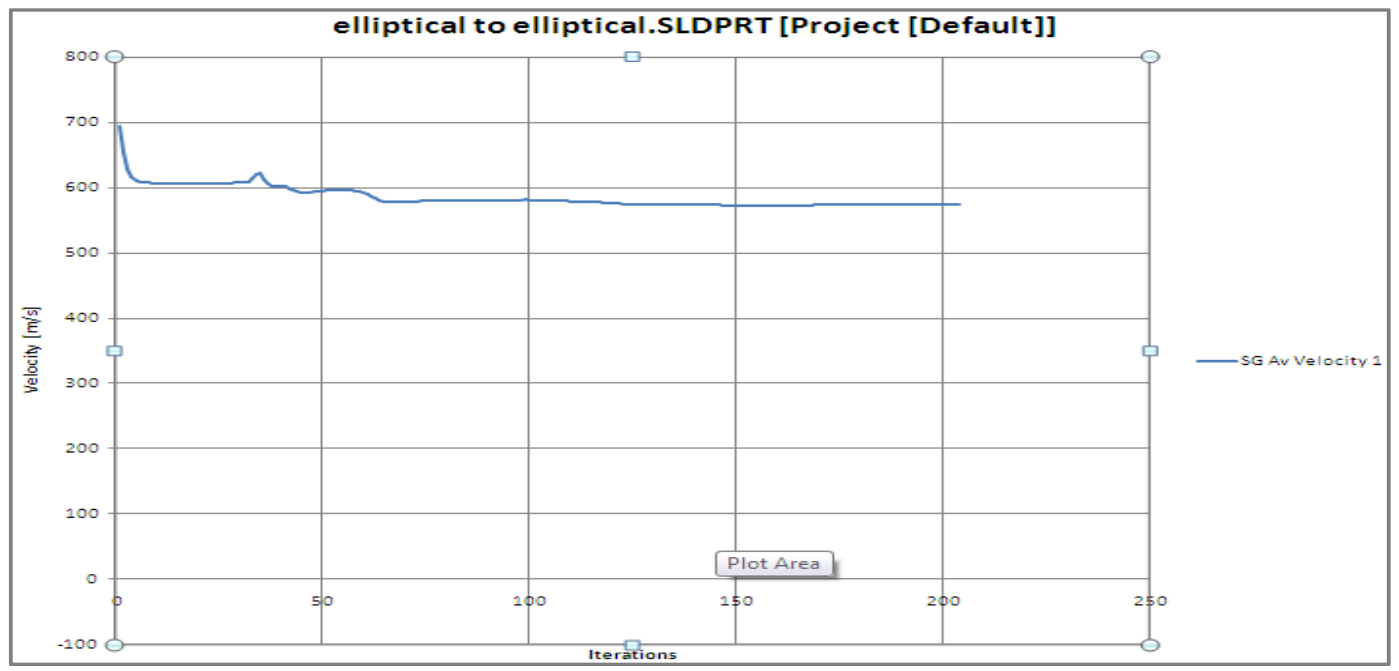

Figure 17: Elliptical nozzle the velocity was taken with iteration

Graph for Elliptical Nozzle-Volume Flow Rate: The graph has been plotted between volume flow rate $\mathrm{m}^{3} / \mathrm{s}$ and number of iteration (figure 18). The graph shows that the volume flow rate increases from -0.000315 to $0.00019 \mathrm{~m}^{3} / \mathrm{s}$ with increase of number of iteration from 0 to 210 in elliptical nozzle of AJM process.

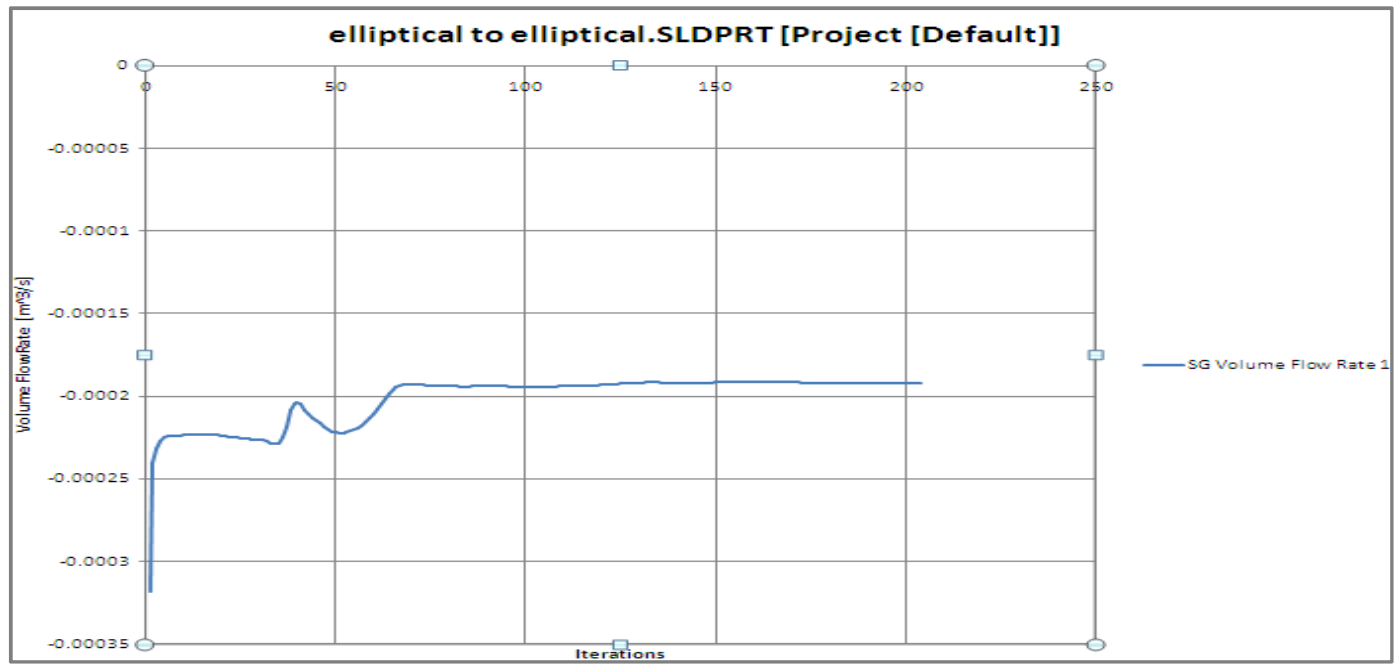

Figure 18: Elliptical nozzle the volume flow rate was taken with iteration

Graph for Elliptical Nozzle-Mass Flow Rate: The graph has been plotted between mass flow rate $\mathrm{kg} / \mathrm{s}$ and number of iteration (figure 19). The graph shows that the velocity decrease from 0 to $-710 \mathrm{~kg} / \mathrm{s}$ with increase of number of iteration from 125 to 210 in elliptical nozzle of AJM process.

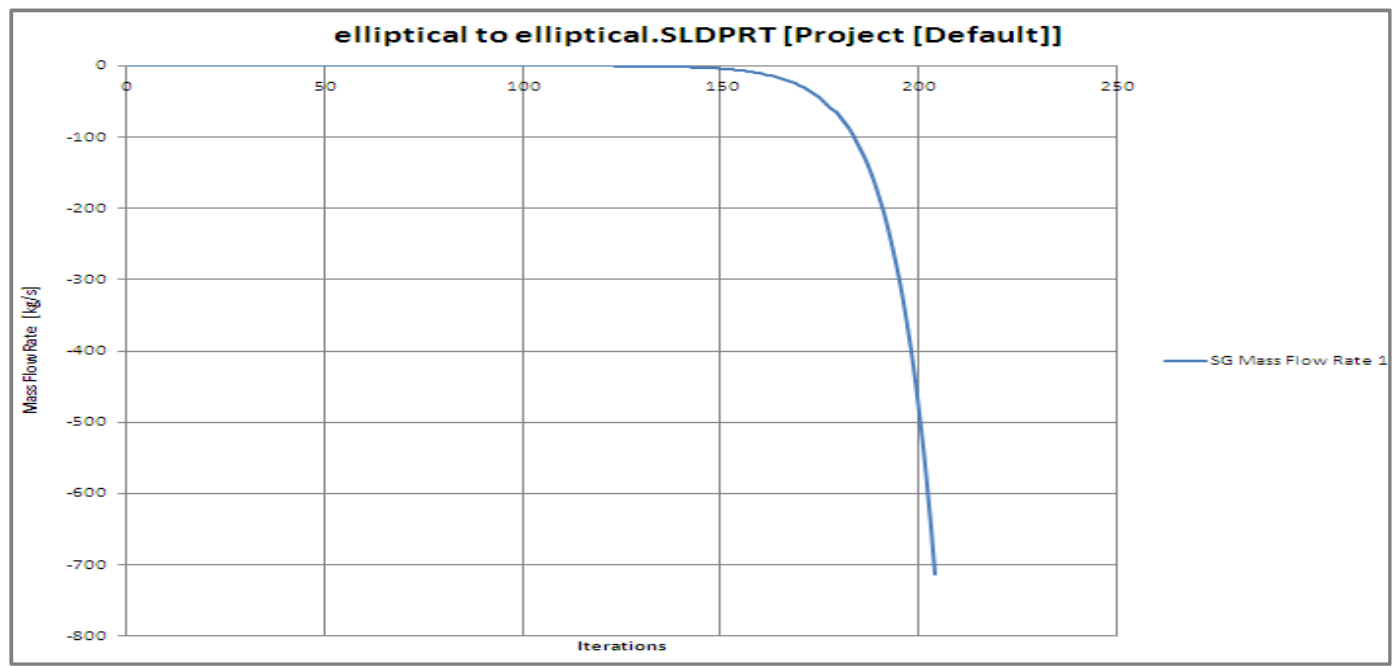

Figure 19: Elliptical nozzle the mass flow rate was taken with iteration 
Baranitharan and Zeelan Basha

Graph for Elliptical Nozzle-Static Pressure: The graph has been plotted between static pressure pa and number of iteration (figure 20). The graph shows that the static
Sci. Technol. Arts Res. J., Jan-March 2015, 4(1): 171-179

pressure from $-1 \mathrm{E}+11$ to $-1.58 \mathrm{E}+11$ pa with increase of number of iteration from 130 to 210 in circular nozzle of AJM process.

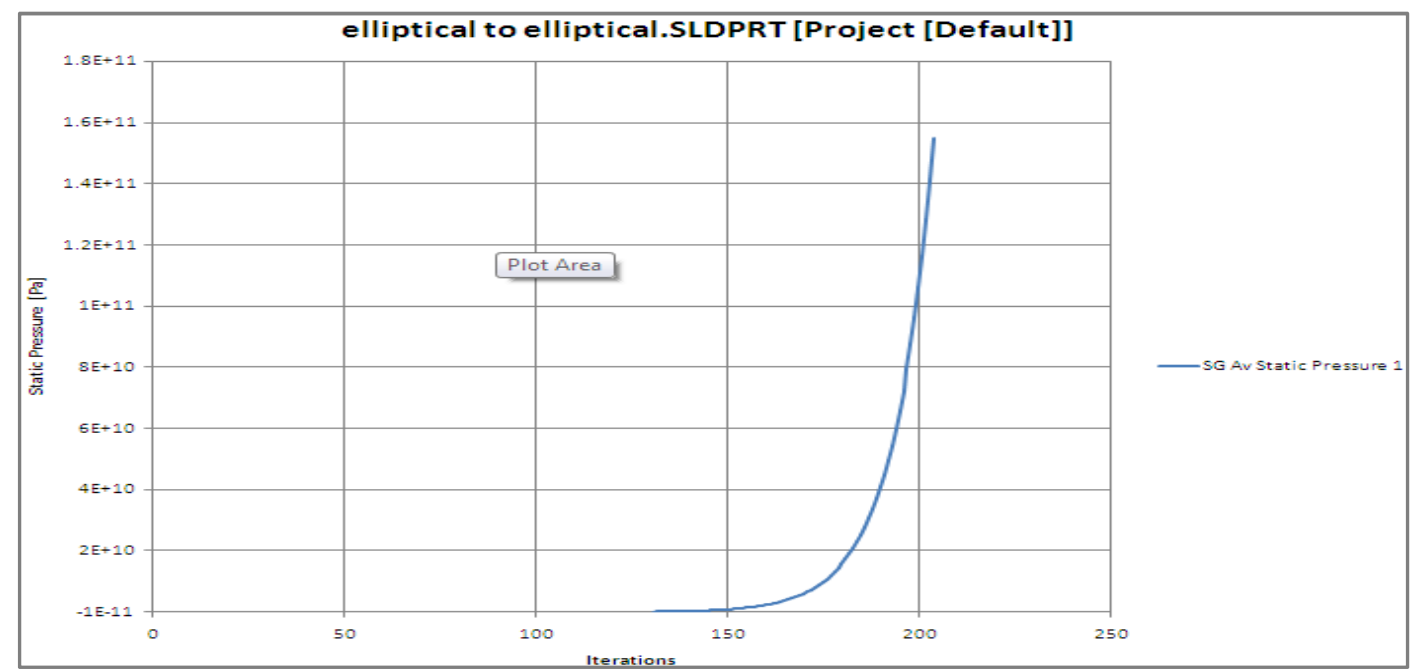

Figure 20: Elliptical nozzle the static pressure was taken with iteration

\section{CONCLUSIONS}

Based on the design and analysis investigation, application of computational fluid dynamics used to analyze improvement of nozzle surface parameters in circular and elliptical nozzle has been discussed. From the result using elliptical nozzle in Abrasive jet Machining following conclusion has been made

- Achieved Improvement upto $5 \%$ of mass flow rate comparing the circular nozzle.

- Improved velocity without affecting any other surface parameters comparing the circular nozzle.

- Material removal rate increases as mass flow increased comparing circular nozzle

- The above advantages and improvement considerably reduces the machining time and improve performance

\section{Conflict of Interest}

Conflict of interest none declared.

\section{REFERENCES}

Alina Oancea., Lorelei Gherman., Laurentiu Slatineanu. And Vasile Braha (2012). Modified Nozzel for Abrasive Jet Engraving. Nonconventional Technologies Review. Romanian Association of Nonconventional Technologies Romania, June, 2012.
Bhaskarchandra and Jagtar Singh (2011). A Study of effect of Process Parameters of Abrasive jet machining. International Journal of Engineering Science and Technology 3(1): 504-513.

Gulhane, U.D., Patkar, P.P., Toraskar Patil, S.P., Patil .A. A. (2013). Analysis of Abrasive Jet Machining Parameters on MRR and Kerf Width of Hard and Brittle Materials Like Ceramic. International Journal of Design and Manufacturing Technology 4(1): 51-58

Olsson, A., Stemme, G., Stemme, E. (1997). Solid State Sensors and Actuators, Transducers 97 Chicago., International Conference 2:1032-1042

Sehgal, K.B. (2011). Study and Optimization of Process Parameters in Abrasive Jet Machining. Impulse 7: 48-52

Umang Anand., Joseph Katz (2003). Prevention of Nozzle Wear in Abrasive Water Suspension Jets (AWSJ) Using Porous Lubricated Nozzles. Journal of Tribology 125:168180

Yan Qusheng (2002). Investigation on the Application of Abrasive Jet Micromachining Design of Nozzle and Machining Experiments. Diamond and Abrasive Engineering 2:194-202. 